\title{
Effects of auditory feedback on a musical performance task*
}

\author{
ANNE GATES $\dagger$ and JOHN L. BRADSHAW \\ Monash University, Clayton, Victoria 3168, Australia
}

\begin{abstract}
Two experiments examined the effects of various feedback conditions on performance by $12 \mathrm{~S}$ on an electronic organ. Playing time scores showed complete absence of auditory feedback to have little or no detrimental effect; in fact, some Ss played fastest under this condition. Delayed auditory feedback (DAF), as expected, caused considerable performance decrement when compared with immediate auditory feedback (IAF). Extraneous prerecorded input as a distractor in the absence of auditory feedback ranked between mixed DAF-with-IAF, and pure DAF, which showed the greatest disruption. The possible role of auditory imagery in musical performance was discussed, together with four components which perhaps contribute to the standard DAF effect: distraction, error repetition, conflict with expectancies and auditory imagery, and presentation of two competing and conflicting signals (IAF and DAF).
\end{abstract}

In normal speech we receive immediate and continuous auditory feedback, transmitted both by air and bone conduction. Delayed auditory feedback (DAF) has been shown to cause disruption to normal speaking (Yates, 1963), and in a qualitatively similar way, to key tapping (Chase, Harvey, Standfast, Rapin, \& Sutton, 1961). The effects of DAF on musical tasks have not been as extensively studied, although music performance has been shown to be disrupted by DAF, both by increased errors in sight reading (Havlicek, 1968) and by longer playing time of a familiar passage (Bradshaw, Nettleton, \& Geffen, 1971, 1972).

The exact nature of the DAF effect is not clear. The delayed feedback may be disruptive in that it interferes with the efficient monitoring of the immediate auditory feedback (IAF). DAF might then have qualitatively similar effects to a complete absence of feedback. Alternatively, the detrimental effect may stem from the disruption caused by a distracting input; DAF might then act in a manner similar to an extraneous input. On the other hand, it may be the discrepancy between the two perceived signals, immediate and delayed, which is disruptive.

In investigating the causes of the DAF effect, performance must be studied in the absence of auditory feedback, under DAF alone, under DAF in the presence of IAF, and with extraneous input instead of auditory feedback. Performance under each of these conditions must be compared with that under normal IAF.

Such manipualtion of auditory feedback in speech is limited by the fact that with nondeaf Ss it is impossible to eliminate completely the immediate, bone-conducted feedback. However, in studying music, the use of the electronic organ enables the $E$ to vary the conditions of

\footnotetext{
*This research was supported by the Music and Psychology Departments of Monash University, and by an award from the Australian Research Grants Committee to the second au thor. We wish to thank Dianne Bradley for her helpful criticisms of the manuscript.

tRequests for reprints should be sent to Anne Gates Psychology Department, Monash University, Clayton, Victoria 3168, Australia.
}

feedback as desired; since the IAF can be completely eliminated, this method permits study of the effects of an absence of auditory feedback and of delayed feedback itself.

Where an absence of feedback has been investigated. effects have differed according to the task employed. Klein and Wolitzsky (1970) reported that under conditions of reduced air-conducted and masked, bone-conducted feedback (produced by $100-\mathrm{dB}$ white noise), speaking or reading speed was no different from, or even faster than performance under normal conditions of feedback. While Ruhm and Cooper (1963) found that the absence of auditory feedback had lit tle or no effect on a rhythmic keytapping task, Chase, Rapin, Gilden, Sutton, and Guilfoyle (1961) found that decreased visual, tactile, or auditory feedback resulted in disruption in a complex, but not in a regular keytapping task. However, Yates (1965b) showed that absence of auditory feedback did not affect accuracy of Morse transmission. An extraneous input has been shown to make little change in speech (Fillenbaum, 1963; Yates, 1965a), perhaps because talking is a grossly overlearned and a socially fundamental skill; indeed, we are continually confronted with the "cocktail party" situation in normal life (Cherry, 1953).

Normally, studies in DAF have investigated the effect in the presence of IAF; however, there is some evidence that the disruption caused by DAF alone may be greater than that produced by DAF-with-IAF. Chase, Sutton, and Rapin (1961) noted that the correct word rate for DAF-with-IAF was higher than for DAF alone (while not mentioning how DAF by itself was achieved), although not as high as for IAF alone. Similarly, Bradshaw et al (1972) demonstrated that, for music performance on an electric organ, "mean playing time with DAF to one ear and IAF to the other $(51.8 \mathrm{sec})$ was almost exactly intermediate between the figure for monaural IAF $(45.4 \mathrm{sec})$ and monaural DAF $(56.6 \mathrm{sec})$ [p. 272]."

One application of the DAF technique has been its 
use in the study of hemispheric specialization (Bradshaw et al, 1971, 1972). Speech was disrupted more when the delayed signal went to the right ear than when it went to the left ear, and conversely in the same paradigm, music performance was disrupted more when the delayed signal -went to the left ear. Such differences in performance were explained in terms of left hemisphere specialization for speech, and right for music (Kimura, 1967).

The following experiments were designed to investigate the effects of different kinds of auditory feedback on music performance. It is by no means certain that the effects would be comparable to those produced in speech, despite similarities between speech and music in terms of temporal patterning and pitch changes. For example, unlike the normal speech situation, a musician, when playing in a group, must attend to the performance of other instrumentalists or singers and vary his own playing accordingly. This difference might therefore become apparent when playing in the presence of an irrelevant musical input. Although DAF in the presence of IAF has been shown to be detrimental to music performance, this study investigates the effects of the delay itself, that is, pure DAF, as well as DAF-with-IAF, and the absence of auditory feedback. It was also asked whether or not the disruptive effects of DAF would indicate a right hemispheric specialization for music, where the Ss play an electronic organ in a situation analogous to speech under DAF, and whether this lateralized DAF condition was more or less detrimental than either no feedback or the usual binaural DAF.

\section{EXPERIMENT I}

The first experiment was designed to ask if auditory feedback is necessary for satisfactory music performance. Is the absence of auditory feedback more or less detrimental than a disruptive feedback (DAF) to music performance? Can laterality differences in the effects of DAF be demonstrated with an electronic organ, where no intensity modulation is possible?

The five conditions studied were (1) IAF, (2) no feedback, (3) DAF to both ears with IAF to both ears, (4) DAF to the right ear with IAF to both ears, and (5) DAF to the left ear with IAF to both ears.

\section{Method}

There were six female and six male paid adult volunteers, all proficient in the playing of a keyboard instrument. None claimed any hearing impairment, and all showed a difference of approximately $5 \mathrm{~dB}$ or less between ears, as measured by a Békésy audiometer (Grason-Stadler Model E800) for a single frequency of $250 \mathrm{~Hz}$; the latter was chosen as it lies in the middle of the range ( \pm one octave) of the piece used in this experiment. All Ss were right-handed with no left-handed close relatives, including grandparents, parents, and siblings.

Apparatus. An electronic organ (National Model SX-88) was used. Output from the organ was channeled to a tape recorder (Revox Model A77) set to give a time delay of $0.18 \mathrm{sec}$ for the
DAF conditions, and to an amplifier (Leak) connected to speakers (Wharfedale Model Linton) for the IAF and DAF conditions. The 0.18 -sec delay interval was chosen because it has been shown to be effective in producing disruption to a music performance (Gates et al, 1974); although it was not the delay causing maximal disruption, the tape recorder giving that delay was not as suitable for the purposes of this experiment. Each $\mathrm{S}$ wore headphones (Maico Auraldomes fitted with TDH 39 earphones); in the conditions in which the DAF went to a single ear, a switch directed the DAF to either one or the other earphone. In the no feedback condition, the amplifier was turned off so that the $S$ could not hear anything of his performance; however, output from the organ to the tape recorder still allowed the $\mathrm{E}$ to monitor S's performance through separate earphones while recording S's playing. Levels for all DAF conditions were set with a Bruel and Kjaer precision sound-level meter (Type 2203) so that at the ear the signal was equal in intensity to that of the external IAF transmitted across the headphones. This procedure took into account the fact that the headphones attenuate sounds in the range 250 to $2,000 \mathrm{~Hz}$ by $20 \mathrm{~dB}$.

IAF was used in all three DAF conditions to simulate speech under DAF, where $S$ hears his voice immediately by air and by bone conduction through both ears as well as delayed, through the earphones.

Material. The test piece was an adaptation of an organ study by John Stainer ("Trio," in The Organ, Novello, no year of publication given). Because it was felt that the parts for each hand should be equal in note length, rhythm, and difficulty, notes of the original score were occasionally omitted, transposed an octave, or lengthened or shortened to attain this end; all changes were within the harmonic and rhythmic constraints of the music. In order to ensure further the equality of the hands, the part played by each hand was transposed an octave, either up or down, so that the part played by the right hand previously was played by the left hand, and vice versa. The original and the transposition were transcribed, and played as one piece.

Procedure. Each $S$ first practiced the piece under normal conditions of air-conducted immediate feedback until he could play it through fluently. He then read typed instructions which described the five kinds of feedback, and which emphasized the need to play as quickly and as accurately as possible. The five conditions were each tested four times on one of four counterbalanced orders. The no feedback condition came midway in each block of five experimental conditions to allow comparison between the IAF and DAF-with-IAF conditions, and between the lateralized DAF conditions. Total playing time was used as a measure of performance. It is the easiest to obtain, particularly with music, and has been used in this context (Bradshaw et al, 1971, 1972; Gates, Bradshaw, \& Nettleton, 1974), as well as for speech (Bradshaw et al, 1971, 1972; Fairbanks, 1955; Timmons, 1971). A time score was found to be more sensitive than an error score in a key tapping task, which is perhaps analogous to music (Rapin, Costa, Mandel, \& Fromowitz, 1966), and also more sensitive than an intensity measure (Karlovich \& Graham, 1966). Playing time has been shown to be only slightly less powerful than correct note rate as a measure for differentiating between IAF and DAF conditions (Gates \& Bradshaw ${ }^{1}$ ).

\section{Results and Discussion}

An analysis of variance revealed no significant effect for sex, although the female Ss tended to play the test piece faster, $F(1,10)=4.6$. There was a highly significant difference between the feedback conditions, $F(4,40)=$ $14.94, \mathrm{p}<.001$. Because the difference between sexes was not significant, the results of both groups were collapsed and analyzed together, using the 
Table 1

Summary of Tests on the Means Using Newman-Keuls Procedure: Experiment I

\begin{tabular}{llllll} 
& \multicolumn{5}{c}{ Conditions } \\
\cline { 2 - 6 } & IAF & $\begin{array}{c}\text { No } \\
\text { Feed- } \\
\text { back }\end{array}$ & $\begin{array}{c}\text { DAF } \\
\text { Right } \\
\text { Ear }\end{array}$ & $\begin{array}{c}\text { DAF } \\
\text { Left } \\
\text { Ear }\end{array}$ & $\begin{array}{c}\text { DAF } \\
\text { Both } \\
\text { Ears }\end{array}$ \\
\hline $\begin{array}{l}\text { Ordered } \\
\text { Means } \dagger\end{array}$ & 53.9 & 54.7 & 57.6 & 58.1 & 58.6 \\
\hline
\end{tabular}

Note-Conditions underlined by a common line do not differ from each other; conditions not underlined by a common line differ significantly at the $p<.01$ level.

tTime in seconds.

Newman-Keuls procedure (Winer, 1971). A summary of the results of tests on the means is given in Table 1.

Performance under the no feedback condition was not statistically different from that under IAF. It is possible that some kind of auditory imagery can guide $\mathrm{Ss}^{\prime}$ responses in the absence of real auditory feedback.

All three of the delay conditions differ considerably $(p<.01)$ from both the IAF and no feedback conditions. The difference between the lateralized DAF conditions, although in the expected direction, failed to reach significance, in spite of the fact that 9 of the $12 \mathrm{Ss}$ showed slower performance with DAF to the left ear.

\section{EXPERIMENT II}

Experiment I has shown that the no feedback condition was similar to IAF, and that both were different from the DAF conditions. Experiment II was designed to ask whether or not the presence of an extraneous, prerecorded input, in the absence of auditory feedback is more disruptive to performance than no input at all. If this extraneous input is a disruptor, is it as powerful a disruptor as DAF? Is the DAF effect due to a discrepancy between the immediate and the delayed signals, or to the delay itself?

The five conditions studied were (1) IAF baseline, (2) no auditory feedback, (3) extraneous prerecorded input (melody with accompaniment on the same instrument and in the same range as the test piece) in the absence of auditory feedback, (4) DAF alone, and (5) DAF-with-IAF.

\section{Method}

Ss were six female and six male paid adult volunteers; half of each group had been $S s$ in Experiment $I$, and the remainder met the same requirements. The same equipment was used as previously, with two exceptions: a second tape recorder (Tandberg 11) was used to deliver the extraneous input to $S$; the switch used to direct input to one or the other earphone was not needed. The test piece and the procedure were the same as before. The IAF condition came midway in each block of five experimental conditions to permit comparison between the DAF conditions, and between the conditions of no feedback and extraneous input with no feedback.

\section{Results and Discussion}

An analysis of variance again revealed no significant effect of sex, $F(1,10)=0.11$. There was a highly significant difference between the feedback conditions, $F(4,40)=11.17, p<.001$. As before, results were analyzed using the Newman-Keuls procedure (Winer, 1971). A summary of tests on the means is given in Table 2.

As before, there was no significant difference between the IAF and no feedback conditions, but a significant difference $(p<.01)$ between the IAF and the DAF conditions; however, there was no difference between the no feedback and DAF-with-IAF condition.

An extraneous, prerecorded input was more disruptive to performance than no auditory feedback $(p<.05)$; in fact, the extraneous condition was more similar to the DAF condition, being intermediate between pure DAF and DAF-with-IAF.

\section{GENERAL DISCUSSION}

Two experiments examined the effects of various kinds of auditory feedback on musical performance. No sex differences were found in the way Ss reacted to different kinds of auditory feedback. In both experiments, performances under IAF and no feedback conditions did not differ statistically. If some form of auditory imagery is employed by the $S$ in controlling his playing, its assistance is suggested by the fact that some Ss played faster under this condition than under the normal IAF condition. Another explanation for faster performance under the no feedback condition could be that immediate feedback provides tempo and error information which could slow performance.

As expected, DAF-with-IAF caused worse performance than IAF alone. Although the increase in playing time may not be as great as is often demonstrated in speech DAF studies, it must be borne in mind that with the electronic organ there remains a considerable degree of immediate proprioceptive feedback to help regulate performance, as well as immediate visual feedback not available in a speech situation. A generally faster performance in the second experiment was found in the DAF-with-IAF and the IAF conditions. However, a comparison of the conditions

Table 2

Summary of Tests on the Means Using Newman-Keuls Procedure: Experiment II

\begin{tabular}{|c|c|c|c|c|c|}
\hline Conditions & IAF & $\begin{array}{l}\text { No } \\
\text { Feed- } \\
\text { back }\end{array}$ & $\begin{array}{c}\text { DAF } \\
+ \\
\text { IAF }\end{array}$ & $\begin{array}{l}\text { Extra- } \\
\text { neous }\end{array}$ & DAF \\
\hline \multirow{2}{*}{$\begin{array}{c}\text { Ordered } \\
\text { Means } \\
\text { (Time in sec) }\end{array}$} & 39.1 & 40.4 & 42.9 & 43.4 & 44.4 \\
\hline & \multicolumn{5}{|c|}{$\overline{-}-\cdots-\cdots-\cdots$} \\
\hline
\end{tabular}

Note-Conaitions underlined by a common line do not differ from each other; conditions not underlined by a common line differ significantly at the $p<.01$ level; conditions underlined by a dotted line differ significantly at the $p<.05$ level. 
shows that a similar ratio increase is maintained between them in both experiments (9\% in the first, and $10 \%$ in the second).

In spite of the fact that DAF-with-IAF was worse than no feedback in Experiment $I$, no significant effect was found in Experiment II. A comparison of the mean playing times of individual Ss shows that six Ss from Experiment I performed under the no feedback condition as well as or better than under IAF, compared with only three Ss from Experiment II who were facilitated by no feedback. All those Ss who participated in both experiments showed similar effects between comparable conditions in the two experiments.

In the laterality comparison, DAF to the left ear with IAF to both ears tended to be worse than DAF to the right ear with IAF to both, this being the case for $75 \%$ of the Ss, though the effect failed to reach significance. This failure may be due to the use of an electronic organ rather than a piano as was used previously by Bradshaw et al (1971) for DAF-with-IAF. Indeed, one of the most common disturbances of performance under DAF in speech (Yates, 1963) and in music (Havlicek, 1968) is increased intensity and misplaced accents, both of which cannot occur on the organ when volume controls are set constant. However, Ss did show changes similar to those reported: repeated notes, decreasing tempo, and pauses. Moreover, Bryden and Allard (1973) have pointed out that of the approximately $95 \%$ of right-handed people who are left-hemisphere dominant for speech, only $80 \%$ to $85 \%$ show a right-ear superiority in dichotic listening. The left-ear effect has not been consistently found, though it has been noted previously for melodic recognition (Kimura, 1964; Spellacy, 1970; Spreen, Spellacy, \& Reid, 1970) and in performance (humming-Kimura, 1967; tapping-Bakker, 1970; and playing music-Bradshaw et al, 1971, 1972).

Pure DAF was significantly worse than both IAF and no feedback, but not different from DAF-with-IAF. These comparisons suggest that the normal DAF (i.e., DAF-with-IAF) effect, as in speech or piano playing, is not solely due to the discrepancy between the immediate and the delayed signals. Indeed, in a similar musical task (Gates, Bradshaw, \& Nettleton, 1974), some Ss were speeded by DAF, suggesting that it is possible to adopt a playing strategy or tempo which maximizes the coincidence of arrival of the delayed note with the playing of another note. The resulting simultaneity is more acceptable than the alternation of immediate and delayed signals, both at the harmonic level and because it avoids the sensation of playing slightly behind the beat. This emphasizes the role of DAF in disrupting the normal rhythmic sequences.

If a form of auditory imagery is thought to operate in the no feedback condition, it could also appear in the pure DAF condition. This would then become functionally equivalent to the DAF-with-IAF condition. Neither DAF alone nor DAF-with-IAF differed significantly from extraneous input. While this demonstrates how DAF may act partly as a distractor, greater disruption from the pure DAF condition as compared with the extraneous condition was evident with all but two Ss. Consequently, the DAF effect cannot be due solely to the influence of a distracting influence as such.

The extraneous condition was significantly worse than both IAF and no feedback. Such an irrelavant input may be considered distracting, simply because it competes for the performer's attention, blocking both external feedback and any internal auditory imagery which might act as a guide. The continued disruptive effect from long DAF delays (in excess of $1 \mathrm{sec}-$ Gates et al, 1974) emphasize the part played by DAF as a distractor.

In conclusion, immediate feedback seems potentially replaceable, at least to some extent, by a form of auditory imagery in the no feedback situation. The latter has the advantage of not permitting errors to disturb S's performance further. DAF disruption seems to stem from several sources. It distracts, in a manner similar to an extraneous input, it repeats an immediately prior error or inadequacy, further disturbing playing, it conflicts with S's previously established expectancies of what he should hear while playing and with any interval auditory imagery, and finally, in conjunction with IAF, it results in the presentation of two discrepant signals, the immediate and the delayed. While the DAF effect is notoriously resistant to any reduction with practice (Yates, 1963), a practice effect, if found, could operate at any of these four levels. Further experiments should aim to study the independent action of the above four components, and the extent to which any of them are differentially reduced with long exposure. In particular, since the tempo of music is more amenable to external experimental manipulation than that of speech, the effect of DAF should be studied in this context (cf. Robinson, 1972, who externally paced the speech of Ss under DAF), with errors or changes as the dependent variable.

\section{REFERENCES}

Bakker, D. J Ear-asymmetry with monaural stimulation: Relations to lateral dominance and lateral awareness. Neuropsychologia, 1970, 8, 103-117.

Bradshaw, J. L., Nettleton, N. C., \& Geffen, G. Ear differences and delayed auditory feedback: Effects on a speech and a music task. Journal of Experimental Psychology, 1971, 91, 85-92.

Bradshaw, J. L., Nettleton, N. C., \& Geffen, G. Ear asymmetry and delayed auditory feedback: Effects of task requirements and competitive stimulation. Journal of Experimental Psychology, 1972, 94, 269-275.

Bryden, M. P., \& Allard, F. Dichotic listening and the development of linguistic processes. University of Waterloo Research Reports in Psychology, January 24, 1973. No. 44.

Chase, R. A., Harvey, S., Standfast, S., Rapin, I., \& Sutton, S. Studies on sensory feedback: I. Effect of delayed auditory feedback on speech and keytapping. Quarterly Journal of Experimental Psychology, 1961, 13, 141-152.

Chase, R. A., Rapin, I., Gilden, L., Sutton, S., \& Guilfoyle, G. Sensory feedback influences on keytapping motor tasks. Quarterly Journal of Experimental Psychology, 1961, 13, 153-167.

Chase, R. A., Sutton, S., \& Rapin, I. Sensory feedback influences on $\mathrm{m}$ otor performance. Journal of Auditory Research, 1961 1, 212-223. 
Cherry, C. Some experiments on the reception of speech with one and with two ears. Journal of the Acoustical Society of America, 1953, 25, 975-979.

Fairbanks, G. Selective vocal effects of delayed auditory feedback. Journal of Speech \& Hearing Disorders, 1955, 20. 333-345.

Fillenbaum, S. Impairment in performance with delayed auditory feedback as related to task characteristics. Journal of Verbal Learning \& Verbal Behavior, 1963, 2, 136-141.

Gates, A., Bradshaw, J. L., \& Nettleton, N. C. Effect of different delayed auditory feedback intervals on a music performance task. Perception \& Psychophysics, 1974, 15, 21-25.

Havlicek. L. L. Effect of delayed auditory feedback on musical performance. Journal of Research in Music Education, 1968, $16,308-318$.

Karlovich, R. S., \& Graham, J. T. Effects of pure tone synchronous and delayed auditory feedback on keytapping performance to a programmed visual stimulus Journal of Speech \& Hearing Research, 1966, 9, 596-603.

Kimura, D. Left-right differences in the perception of melodies. Quarterly Journal of Experimental Psychology, 1964, 16, 355-358.

Kimura, D. Functional asymmetry of the brain in dichotic listening. Cortex, 1967, 3, 163-178.

Klein, G. S., \& Wolitzky, D. L. Vocal isolation: Effects of occluding auditory feedback from one's own voice. Journal of Abnormal Psychology, 1970, 75, 50-56.

Rapin. I., Costa, L, D., Mandel, I. J., \& Fromowitz, A. J. Keytapping and delayed feedback. Journal of Speech \& Hearing Research, 1966, 9, 278-288.

Robinson, G. M. The delayed auditory feedback effect is a function of speech rate. Journal of Experimental Psychology. $1972,95,1-5$.

Ruhm, H. B., \& Cooper, W. A.. Jr. Some factors that influence pure tone delayed auditory feedback. Journal of Speech \& Hearing Research, 1963, 6, 22 3-237.

Spellacy, $F$. Lateral preferences in the identification of pattern stimuli. Journal of the Acoustical Society of America, 1970 47, 574-578.

Spreen, O., Spellacy, F. J., \& Reid, J. R. The effect of interstimulus interval and intensity on ear asymmetry for non verbal stimuli in dichotic listening. Neuropsychologia, 1970 . $8,245-250$.

Timmons, B. A. Sex as a factor influencing sensitivity to delayed auditory feedback. Perceptual \& Motor Skills, 1971, 32 $824-826$.

Winer. B. J. Statistical principles in experimental design. (2nd ed.) New York: McGraw-Hill, 1971.

Yates, A. J. Delayed auditory feedback. Psychological Bulletin, $1963,60,213-232$.

Yates, A. J. Delayed auditory feedback and shadowing. Quarterly Journal of Experimental Psychology, 1965a, 17, 125-131.

Yates, A. J. Effects of delayed auditory feedback on Morse transmission by skilled operators. Journal of Experimental Psychology, 1965b, 69, 467-475.

\section{NOTE}

1. Gates, A., \& Bradshaw, J. L. Interactions of ears, hands, and cerebral hemispheres in a musical perform ance task requiring bimanual coordination. In preparation, 1974.

(Received for publication August 13, 1973; revision accepted March 21,1974 .) 